\title{
Pion physics in two flavor strong coupling lattice QED
}

\section{J. Cecile*}

Department of Physics, Duke University, Durham, NC 27708, USA

E-mail: djcecile@phy.duke.edu

\begin{abstract}
We consider the lattice field theory involving two flavors of staggered quarks which interact with $U(1)$ gauge fields in the strong coupling limit. For massless quarks, this theory has an $S U_{L}(2) \times$ $S U_{R}(2) \times U_{A}(1)$ symmetry. We show explicitly how pions emerge through the phenomena of confinement in this theory. We also show how one can incorporate the physics of the anomaly in this theory. Thus, our approach is a good pedagogical tool to explain how pions arise in real QCD. Another advantage of our approach is that we can easily design efficient cluster algorithms to compute a variety of quantities close to the chiral limit, thus allowing us to understand the low energy physics in a QCD-like setting from first principles.
\end{abstract}

XXIVth International Symposium on Lattice Field Theory

July 23-28, 2006

Tucson, Arizona, USA

${ }^{*}$ Speaker. 


\section{Introduction}

One of the outstanding problems in lattice QCD is to compute low energy hadronic observables, which are dominated by the physics of light quarks, with controlled errors. Although progress is being made in terms of fermion algorithms for lattice QCD, it will still be diffi cult to approach realistic quark masses in the near future. As a result, calculations are performed at unphysically large quark masses and then extrapolations to realistic quark masses are performed using chiral perturbation theory. However, for such an approach to be reliable, we must know the range over which the chiral expansion is valid. Unfortunately, this is not very well understood. In fact a systematic study of chiral perturbation theory as an effective fi eld theory that describes a more fundamental lattice fi eld theory has not been attempted in many situations. Thus, it is useful to study a simpler lattice fi eld theory with the same symmetries of lattice QCD and make connections with chiral perturbation theory. This is the main motivation for our work.

We study the physics of strongly coupled lattice QED with two flavors of staggered fermions which we refer to as our model throughout this manuscript. Although this model does not describe full QCD, it is an interesting model which has the same symmetries of two flavor QCD and was recently used to study the chiral phase transition [1]. Note that even a $U(1)$ gauge theory exhibits confi nement in the strong coupling limit, and the taste symmetry is irrelevant since it is maximally broken at strong couplings. Our model allows us to study the pion physics from a fundamental lattice fi eld theory, very similar to QCD, so that we can understand the usefulness of chiral perturbation theory as an effective description of low energy physics. It is important to note that unless we fi nd a way to fi ne tune our model we will be dominated by lattice artifacts since at strong coupling the pion decay constant $F_{\pi}$ is naturally close to the cutoff. In order to circumvent this problem, we defi ne our model in $d+1$ dimensions where $d=4$ is the space time dimensions. The extra dimension plays the role of a fi ctitious temperature which allows us to tune to a critical point where $F_{\pi}$ will be much smaller than the cutoff. Thus, we can still explore the physics of a continuum limit even in the strong coupling limit.

The motivation for studying a strongly coupled theory is that the gauge dependent degrees of freedom can be integrated over, which signifi cantly simplifi es the theory. Further, a new class of algorithms, the Directed Path Algorithm, has recently been designed for strongly coupled gauge theories, by which it is possible to study the chiral limit very effi ciently [2]. We have extended this algorithm to our model and are currently testing it. Here we report on current progress.

We also wish to compute via Lüscher's method [3] quantities that are measurable in scattering experiments. Specifi cally, we would like to measure scattering lengths, characterize and understand resonances which may exist, and provide a setting to understand non-perturbative features that can arise in a QCD like theory and that go beyond the scope of chiral perturbation theory. Such a study appears impossible with the current technology of lattice QCD since some physical processes which occur for suffi ciently light quarks, e.g. the decay of the $\rho$ meson into two pions, is forbidden at the quark masses currently used.

\section{Model and Symmetries}

The Euclidean space action of the $N_{f}=2$ QED model we consider is given by (Note that the 
usual factors $\frac{1}{2}$ are absorbed into the fi eld defi nitions):

$$
S=-\sum_{x, \mu} \eta_{\mu, x}\left[\mathrm{e}^{i \phi_{\mu, x}} \bar{\psi}_{x} \psi_{x+\hat{\mu}}-\mathrm{e}^{-i \phi_{\mu, x}} \bar{\psi}_{x+\hat{\mu}} \psi_{x}\right]-\sum_{x}\left[m \bar{\psi}_{x} \psi_{x}+\frac{\tilde{c}}{2}\left(\bar{\psi}_{x} \psi_{x}\right)^{2}\right]
$$

where $x$ denotes a lattice site on a $d+1$ dimensional hypercubic lattice $L_{t} \times L^{d}$. $\bar{\psi}_{x}$ and $\psi_{x}$ are two component Grassman fi elds that represent the two quark flavors of mass $m$, and $\phi_{\mu, x}$ is the $U(1)$ gauge fi eld through which the fi elds interact. Note that $\mu$ runs over the temporal and spatial directions $0,1,2, \ldots, d$ with 0 denoting the temporal direction. The usual staggered fermion phase factors $\eta_{\mu, x}$ obey the relations: $\eta_{0, x}^{2}=T$ and $\eta_{i, x}^{2}=1$ for $i=1,2, \ldots, d$. The parameter $T$ is the fi ctitious temperature which will be used to the continuum limit. The coupling $\tilde{c}$ will set the strength of the anomaly.

We now discuss how our model has the same symmetries and symmetry breaking patterns of full QCD. It is fi rst useful to note that any sum over lattice sites can be decomposed into a sum over even and $o d d$ sites. At $\tilde{c}, m=0$, the action exhibits a global $S U(2) \times S U(2) \times U_{A}(1)$ symmetry. In particular, the action is invariant under the following $U_{A}(1)$ and $S U_{L}(2)$ transformations (respectively):

$$
\begin{array}{rr}
\bar{\psi}_{o} \rightarrow \bar{\psi}_{o} \exp (i \theta) & \psi_{o} \rightarrow \exp (i \theta) \psi_{o} \\
\bar{\psi}_{e} \rightarrow \bar{\psi}_{e} \exp (-i \theta) & \psi_{e} \rightarrow \exp (-i \theta) \psi_{e} \\
\bar{\psi}_{o} \rightarrow \bar{\psi}_{o} V_{L}^{\dagger} & \psi_{o} \rightarrow \psi_{o} \\
\bar{\psi}_{e} \rightarrow \bar{\psi}_{e} & \psi_{e} \rightarrow V_{L} \psi_{e}
\end{array}
$$

$S U_{R}(2)$ is obtained by $V_{L} \Leftrightarrow V_{R}$ and $o \Leftrightarrow e$. Here $V_{L}$ and $V_{R}$ are $S U(2)$ matrices and can be parameterized by: $\exp (i \vec{\theta} \cdot \vec{\sigma})$ where $\sigma_{i}$ is a Pauli matrix that acts on the flavor space. At $\tilde{c} \neq 0, U_{A}(1)$ is explicitly broken and the action is invariant under $S U_{L}(2) \times S U_{R}(2) \times Z_{2}$. Thus, the coupling $\tilde{c}$ induces the effects of the anomaly. Further at $m \neq 0$, it is necessary to set $V_{L}=V_{R}$ for the action to remain invariant. Thus, with a mass term the chiral symmetry $S U_{L}(2) \times S U_{R}(2)$ is explicitly broken down to $S U_{V}(2)$. In order to mimic QCD we need to set $\tilde{c} \neq 0$ and $m \neq 0$. Hence, our model has the same chiral symmetry as full QCD. Further, based on previous mean fi eld strong coupling calculations [4], we expect that the symmetry breaking pattern is also similar to full QCD.

\section{Mapping to a Monomer - Dimer - Pion Loop - Instanton Model}

The partition function of our model is equivalent to that of a classical statistical mechanics model involving confi gurations made up of gauge invariant objects such as monomers, dimers, pion loops and instantons [5]. We denote these as MDPI configurations. Note that a double monomer on a site breaks the $U_{A}(1)$ symmetry but not the $S U_{L}(2) \times S U_{R}(2)$ symmetry and hence is called an instanton. In addition to these, each confi guration can contain open loops of dimers which terminate on monomers, closed loop of dimers and oriented closed pion loops made up of oriented dimers. Explicitly, the partition function is given by:

$$
Z=\sum_{\left[I, n^{d}, n^{u}, \pi_{\mu}^{d}, \pi_{\mu}^{u}, \pi_{\mu}^{1}\right]} \prod_{x, \mu} m^{n_{d}(x)} m^{n_{u}(x)} c^{I(x)}
$$


where $\left[I, n^{d}, n^{u}, \pi_{\mu}^{d}, \pi_{\mu}^{u}, \pi_{\mu}^{1}\right]$ denotes a MDPI confi guration. Note $I(x)$ is the number of instantons on a site $x, n_{d}(x)$ the number of $d$ monomers, $n_{u}(x)$ the number of $u$ monomers, $\pi_{\mu}^{d}$ the number of $d$ dimers, $\pi_{\mu}^{u}$ the number of $u$ dimers, and $\pi_{\mu}^{1}$ the number of oriented $\bar{u} d$ or $\bar{d} u$ dimers. The allowed values are:

$$
I(x)=0,2, \quad n_{d}(x)=0,1 \quad n_{u}(x)=0,1 \quad \pi_{\mu}^{d}(x)=0,1 \quad \pi_{\mu}^{u}(x)=0,1 \quad \pi_{\mu}^{1}(x)=-1,0,1
$$

Due to the Grassmann nature of the observables the following constraints must also be satisfi ed:

$$
\begin{aligned}
\sum_{\mu} \pi_{\mu}^{1}(x) & =0 \\
I(x)+\sum_{\mu}\left[\pi_{\mu}^{u}(x)+\pi_{\mu}^{d}(x)+n^{u}(x)+n^{d}(x)\right]+\sum_{\mu}\left|\pi_{\mu}^{1}(x)\right| & =2 \\
n_{u}(x)+\sum_{\mu}\left[\pi_{\mu}^{u}(x)-\pi_{\mu}^{d}(x)\right]-n_{d}(x) & =0
\end{aligned}
$$

Figure 1 gives an illustration of an MDPI confi guration in $1+1$ dimensions.

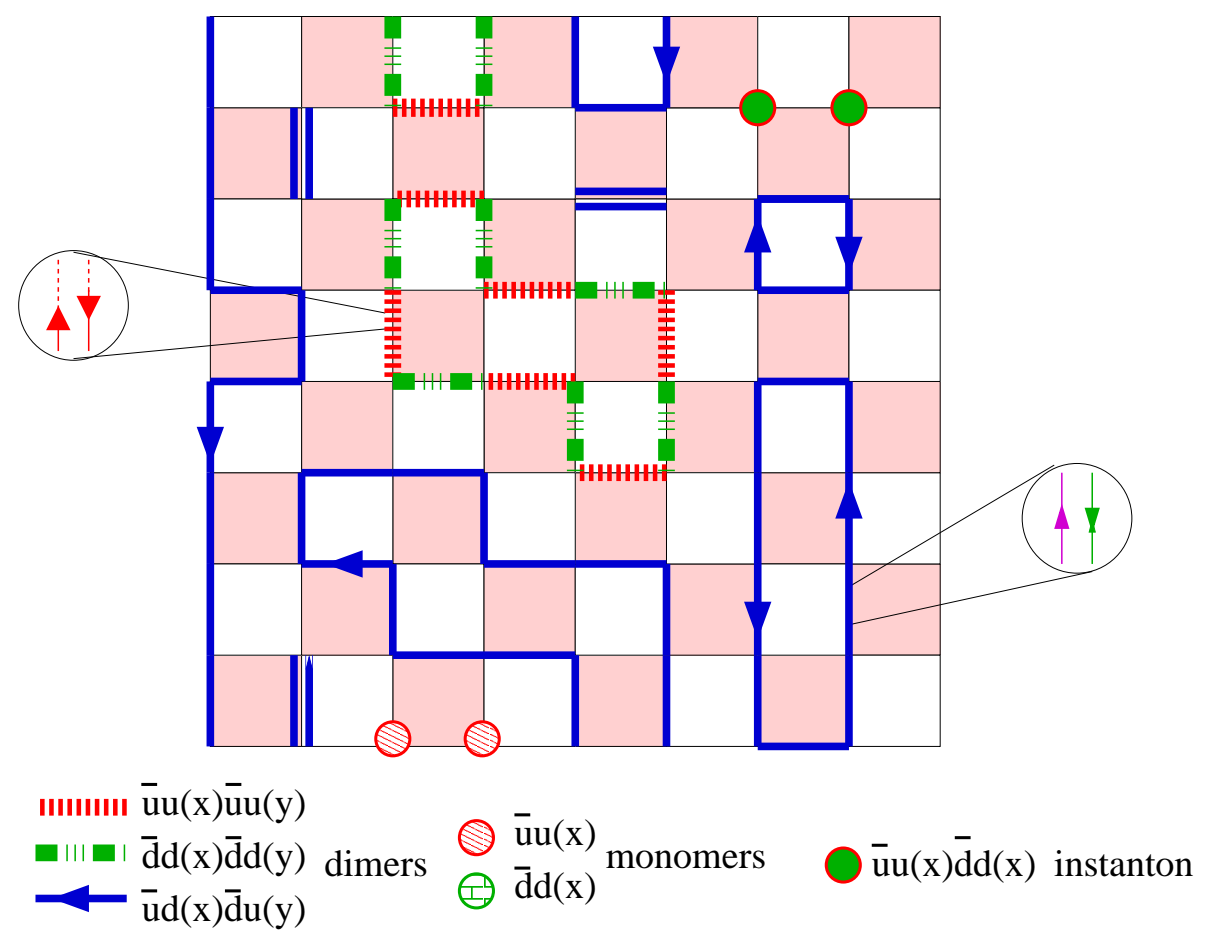

Figure 1: An example of a $2 \times 2$ lattice configuration as discussed in the text.

\section{Algorithm and Observables}

A Directed Path Algorithm can be constructed to update the MDPI confi gurations. Our algorithm is an extension of the algorithms discussed in $[2,6]$. We have three update routines: 
1. A loop flip is an operation in which the orientation of a pion loop or a dimer loop is changed. Note that each dimer loop also contains two states.

2. A loop swap which flips a dimer loop to a pion loop and vice versa.

3. An update which creates and destroys instantons, double bonds, dimer loops, and pion loops. This is similar to the directed loop update constructed in $[2,6]$ and will be discussed in detail elsewhere.

Numerous observables can be measured with this algorithm. The simplest are the three helicity moduli or current susceptibilities. In particular, for a conserved current $J_{\mu}^{i}(x)$, the helicity modulus (current susceptibility) is defi ned as:

$$
Y_{w}^{i}=\frac{1}{d L^{d}}\left\langle\sum_{\mu=1}^{d}\left(\sum_{x} J_{\mu}^{i}(x)\right)^{2}\right\rangle
$$

where we are assuming a $L_{t} \times L^{d}$ lattice. There are three conserved currents in our model. They are the axial, chiral, and vector currents which are given by:

$$
\begin{aligned}
J_{\mu}^{A}(x) & =(-1)^{x}\left[\pi_{\mu}^{u}(x)+\pi_{\mu}^{d}(x)+\left|\pi_{\mu}^{1}(x)\right|\right] \\
J_{\mu}^{C}(x) & =(-1)^{x}\left[\pi_{\mu}^{u}(x)-\pi_{\mu}^{d}(x)\right] \\
J_{\mu}^{V}(x) & =\pi_{\mu}^{1}(x)
\end{aligned}
$$

We also can measure correlation functions of pions defi ned as:

$$
\begin{aligned}
& G_{\pi}(x, y)=\frac{(-1)^{x+y}}{2}\left\langle\bar{\psi}_{x} \sigma^{3} \psi_{x} \bar{\psi}_{y} \sigma^{3} \psi_{y}\right\rangle \\
& G_{\eta}(x, y)=\frac{(-1)^{x+y}}{2}\left\langle\bar{\psi}_{x} \psi_{x} \bar{\psi}_{y} \psi_{y}\right\rangle
\end{aligned}
$$

The corresponding susceptibilities, $\chi_{\pi}$ and $\chi_{\eta}$ are given by:

$$
\chi=\frac{1}{L_{t} L^{d}} \sum_{x, y} G(x, y)
$$

The directed path algorithm allows a straightforward measurement of $G(x, y)$ and $\chi$. Details will be given elsewhere.

\section{Results}

We have compared the measured observables to exact values on $2 \times 2$ lattices and found excellent agreement as shown in Tables 1,2. Below are the analytic expressions for the partition function, the chiral, vector, and axial helicity moduli and the two susceptibilities, defi ned in the 


\begin{tabular}{ccccccccc}
\hline \hline$T$ & $c$ & $m$ & Algo. & Exact & Algo. & Exact & Algo. & Exact \\
\hline & & & $Y_{w}^{A}$ & & $Y_{w}^{C}$ & & $Y_{w}^{V}$ & \\
1.0 & 0.5 & 0.0 & $0.8023(9)$ & $0.80246 \ldots$ & $0.5763(6)$ & $0.57721 \ldots$ & $0.5771(8)$ & $0.57721 \ldots$ \\
1.5 & 0.5 & 0.0 & $0.5212(7)$ & $0.52141 \ldots$ & $0.3275(6)$ & $0.32790 \ldots$ & $0.3274(7)$ & $0.32790 \ldots$ \\
1.0 & 1.0 & 0.0 & $0.7449(8)$ & $0.74534 \ldots$ & $0.5470(7)$ & $0.54658 \ldots$ & $0.5468(8)$ & $0.54658 .$. \\
1.5 & 1.0 & 0.0 & $0.4967(7)$ & $0.49720 \ldots$ & $0.3178(5)$ & $0.31821 \ldots$ & $0.3175(6)$ & $0.31821 \ldots$ \\
1.0 & 1.5 & 0.0 & $0.6667(8)$ & $0.66645 \ldots$ & $0.5016(5)$ & $0.50240 \ldots$ & $0.5014(6)$ & $0.50240 \ldots$ \\
1.5 & 1.5 & 0.0 & $0.4607(6)$ & $0.46147 \ldots$ & $0.3029(5)$ & $0.30325 \ldots$ & $0.3033(6)$ & $0.30325 \ldots$ \\
1.0 & 2.0 & 0.0 & $0.5812(7)$ & $0.58064 \ldots$ & $0.4519(6)$ & $0.45161 \ldots$ & $0.4521(6)$ & $0.45161 \ldots$ \\
1.5 & 2.0 & 0.0 & $0.4199(5)$ & $0.41927 \ldots$ & $0.2853(5)$ & $0.28451 \ldots$ & $0.2853(5)$ & $0.28451 \ldots$ \\
\hline \hline
\end{tabular}

Table 1: Helicity moduli for a $2 \times 2$ lattice as discussed in the text.

\begin{tabular}{ccccccc}
\hline \hline & \multicolumn{3}{c}{$\chi_{\pi}$} & \multicolumn{2}{c}{$\chi_{\eta}$} \\
$T$ & $c$ & $m$ & Algo. & Exact & Algo. & Exact \\
\hline 1.0 & 0.5 & 0.0 & $0.3601(3)$ & $0.359877 \ldots$ & $0.2172(2)$ & $0.217334 \ldots$ \\
1.5 & 0.5 & 0.0 & $0.2676(2)$ & $0.267764 \ldots$ & $0.1824(1)$ & $0.182429 \ldots$ \\
1.0 & 1.0 & 0.0 & $0.4040(3)$ & $0.403727 \ldots$ & $0.1429(2)$ & $0.142857 \ldots$ \\
1.5 & 1.0 & 0.0 & $0.2981(2)$ & $0.298322 \ldots$ & $0.1367(1)$ & $0.136731 \ldots$ \\
1.0 & 1.5 & 0.0 & $0.4220(3)$ & $0.422301 \ldots$ & $0.0801(1)$ & $0.080103 \ldots$ \\
1.5 & 1.5 & 0.0 & $0.3172(3)$ & $0.317264 \ldots$ & $0.0948(1)$ & $0.094767 \ldots$ \\
1.0 & 2.0 & 0.0 & $0.4194(2)$ & $0.419355 \ldots$ & $0.0323(1)$ & $0.032258 \ldots$ \\
1.5 & 2.0 & 0.0 & $0.3251(3)$ & $0.324754 \ldots$ & $0.0588(1)$ & $0.058961 \ldots$ \\
\hline \hline
\end{tabular}

Table 2: Susceptibilities for a $2 \times 2$ lattice as discussed in the text.

previous section, for a $2 \times 2$ lattice (Note that $c=\tilde{c}+m^{2}$ ):

$$
\begin{aligned}
Z(T, c, m) & =36 T^{4}+64 T^{2}+36+c^{4}+12\left(1+T^{2}\right) c^{2}+8(1+T) c^{2} m^{2} \\
& +32 T c m^{2}+16\left(1+T^{2}\right) m^{4}+48\left(1+T^{3}\right) m^{2}+32\left(T+T^{2}\right) m^{2} \\
2 Z \times Y_{W}^{C}(T, c, m) & =96+64 T^{2}+16 c^{2}+112 m^{2}+32 m^{4}+8 c^{2} m^{2}+32 T c m^{2} \\
& +32\left(2 T+T^{2}\right) m^{2} \\
2 Z \times Y_{W}^{V}(T, c, m) & =96+64 T^{2}+16 c^{2}+64 m^{2} \\
Y_{W}^{A}(T, c, m) & =Y_{W}^{C}(T, c, m)+\frac{64 T^{2} m^{2}}{2 Z} \\
2 Z \times \chi_{\pi} & =24 T^{3}+16 T^{2}+16 T+24+4(1+T) c^{2}+4\left(3+4 T+3 T^{2}\right) c+2 c^{3} \\
& +8(1+T) c m^{2}+16\left(1+T+T^{2}\right) m^{2} \\
2 Z \times \chi_{\eta} & =24 T^{3}+16 T^{2}+16 T+24+4\left(1+T+m^{2}\right) c^{2}-4\left(3-4 T+3 T^{2}\right) c \\
& -2 c^{3}-8(1+T) c m^{2}+8\left(5+6 T+5 T^{2}\right) m^{2}+16(1+T) m^{4}
\end{aligned}
$$

The exact analytic results are shown in Tables 1,2 along with values calculated from our algorithm for various $T, c$ and $m$. 


\section{Conclusions and Future Work}

Based on the results in the previous section, we conclude that our algorithm can in principle be used to study $N_{f}=2$ lattice QED. Based on previous experience we expect the algorithm to be effi cient for a range of parameters. We plan to study the model in $d=4$ dimensions and match our results to chiral perturbation theory. We will assume that the lattice cutoff is of the order of $1 \mathrm{GeV}$, and tune $T$ and $c$ so that our $F_{\pi}$ and $m_{\eta}$ are close to their physical values. We then plan to make connections of our data to chiral perturbation theory as we change $m$. Ultimately, we also plan to compute the effects of the quark mass on pion scattering by measuring the appropriate two and four point correlation functions and extracting scattering phase shifts and lengths via Luscher's method as done in [7].

\section{Acknowledgments}

This work was done in collaboration with S.Chandrasekharan. It was partially supported by the DOE grant DE-FG02-05ER41368. The author wishes to thank Fu-Jiun Jiang for useful discussions.

\section{References}

[1] S. Chandrasekharan and A. Mehta, contribution to this proceedings.

[2] D. Adams and S. Chandrasekharan, Nucl. Phys. B 662, 220 (2003).

[3] M. Lüscher, Nucl. Phys. B 354, 351 (1991).

[4] H. Kluberg-Stern, A. Morel, and B. Petersson, Nucl. Phys. B 215, 527 (1983).

[5] P. Rossi, U. Wolff, Nucl.Phys. B 248, 105 (1984).

[6] S. Chandrasekharan and F. Jiang, Phys.Rev. D 74, 014506 (2006).

[7] S. Beane, P. Bedaque, K. Orginos, and M. Savage, Phys. Rev. D 73, 4503 (2006). 